\title{
Die Metropolregionen in der Raumordnungspolitik Deutschlands - ein neues strategisches Raumbild?
}

\section{Hans Heinrich Blotevogel, Duisburg}

\section{Die Fragestellung}

Die sog. «konstruktivistische Wende» hat in vielen Humanwissenschaften und nicht zuletzt auch in der Humangeographie zu einer Veränderung der Forschungsperspektiven geführt. Erstens wurde bewusst, dass Geographinnen und Geographen die Semantik ihres Denkens, Redens, Forschens und Schreibens über «Räume», «Regionen», «Städte» usw. nicht in naiver Form als identisch mit dem Gegenstand selbst annehmen können, sondern dass sie stets mit Repräsentationen operieren. Geographen registrieren zunehmend die semantische Kontingenz der verwendeten raumbezogene Begriffe und reflektieren die Pragmatik ihrer Verwendung sowohl in der Alltags- als auch in der Wissenschaftskommunikation. Sie erzeugen bzw. reproduzieren Repräsentationen der behandelten Objekte in der Form von Texten, Karten, Graphiken usw. und erkennen zunehmend, wie schwankend das positivistische Axiom der objektiven Erfassbarkeit der als vom Beobachter unabhängig existierend angenommenen Realität geworden ist.

Zweitens wurde bewusst, dass diese Repräsentationen nicht einfach harmlose, neutrale Medien der Kommunikation sind, sondern in komplexer Weise in die diskursiven Praktiken und institutionellen Ordnungen der Gesellschaft eingelassen sind. Geographische Repräsentationen sind sowohl Vehikel als auch Ergebnis der Durchsetzung von gesellschaftlichen und wirtschaftlichen Interessen und Macht. Sie strukturieren die Wirklichkeitsdeutungen vor, sie erzeugen und vermitteln Raumbilder, sie implizieren und propagieren Sinnzuweisungen («Heimat», «Nation» usw.), und sie «rahmen» die Diskurse um raumbezogene Politik und Planung. Natürlich sind Geographen nicht die einzigen und bei weitem nicht die wichtigsten (Re-)Konstrukteure geographischer Repräsentationen, aber sie wirken mit, sei es in der Schulgeographie, der Geopolitik, der Landes- und Länderkunde, sei es in der Stadtentwicklung, Regional- und Raumordnungspolitik.

Über die Rolle von raumbezogenen Repräsentationen und Raumbildern in den Politik- und Planungsdiskursen und speziell in der Regional- und Raumordnungspolitik existiert bisher nur eine verhältnismäßig spärliche Literatur. Wichtige Beiträge stammen von der angloame- rikanischen und niederländischen Planungsforschung (Faludi 1996, 1998, Faludi \& van der Falk 1994, Hajer 2000, Pløger 2001, Zonnefeld 2000), während die deutschsprachige Planungsforschung noch weitgehend Fehlanzeige vermeldet (erste Ansätze bei FürST 1997, Grunwald 1996, Ipsen 1997, KNieling 2000). Allerdings bedarf es keiner großen prophetischen Gabe, um vorauszusagen, dass mit dem "cultural turn" in den Humanwissenschaften (LACKNER \& WERNER 1999) die Verwendung von Raumbegriffen und Raumbildern in den Politik- und Planungsdiskursen künftig eine größere Aufmerksamkeit erfahren wird.

Im Folgenden wird der Frage nachgegangen, inwieweit die Einführung der raumordnungspolitischen Kategorie der «Europäischen Metropolregion» (EMR) in die Raumordnungspolitik der Bundesrepublik Deutschland seit 1995 als Einführung eines neuen strategischen Raumbildes zu verstehen ist, mit der eine normative Umorientierung der Raumordnungspolitik verbunden ist. Diskutiert werden soll die These, dass die Einführung der Metropolen-Semantik zu verstehen ist als Reaktion der deutschen Raumordnungspolitik auf eine ganze Reihe von externen Herausforderungen und als Ausdruck einer tiefgreifenden Verschiebung des raumordnungspolitischen Selbstverständnisses.

\section{Hintergründe der aktuellen Diskurse über Metropolen}

Schon ein bis zwei Jahrzehnte vor der Einführung der neuen raumordnungspolitischen Kategorie hatte das Thema «Metropolen» - nicht nur in Deutschland Konjunktur (MATEJovskı 2000). Wo liegen die Gründe für das auffällige Interesse an den vielfältigen aktuellen Metropolen-Diskursen in Politik, Wissenschaft und Öffentlichkeit? Tatsächlich mehren sich seit einiger Zeit in den Humanwissenschaften Symposien, Aufsätze und Bücher zum Thema «Metropolen» (und selbstverständlich ist auch das Themenheft der GEOgraphica Helvetica ein Teil dieser Konjunktur). Die großen Städte und Stadtregionen entdecken ihre mehr oder (häufiger) minder ausgeprägten metropolitanen Funktionen und versuchen, sich im Standortwettbewerb des europäischen oder gar des globalen Systems der großen Städte und Metropolen zu positionieren (Thornley 2000).

Mindestens sieben Hintergrund-Aspekte für die aktuelle Diskurskonjunktur lassen sich identifizieren: 
Erstens die Umwertungen im deutschen Städtesystem als Folge der deutschen Einigung: In den Jahrzehnten der deutschen Teilung hatte sich eine relativ stabile arbeitsteilige Struktur herausgebildet. In der alten Bundesrepublik fungierte Hamburg als Großhandels- und Medienzentrum, Frankfurt als Banken- und Verkehrszentrum, Bonn als Regierungssitz usw. Auch in der DDR standen die großen Städte wie (Ost-)Berlin, Dresden, Leipzig und Rostock in einem System komplementärer Funktionsspezialisierungen. Mit der deutschen Einigung und dem Hauptstadtbeschluss von 1991 geriet dieses Gefüge ins Wanken. Berlin meldete sich mit seinem Anspruch als deutsche Metropole zurück, und seitdem häufen sich die Symposien und Bücher zur Frage, ob, wie und wann Berlin endlich wieder die deutsche Metropole wird. Der Anspruch Berlins blieb nicht ohne Rückwirkungen auf seine Konkurrenten: München fürchtet die Provinzialisierung, Hamburg bündelt seine Kräfte zusammen mit dem Umland zur «Metropolregion», und am Rhein sieht der nordrhein-westfälische Ministerpräsident Clement nach dem Verlust der Bonner Regierungsfunktionen eine angemessene Positionierung eher im nordwesteuropäischen Raum im Konnex mit Brüssel und der Randstad Holland.

Zweitens: Die Integration der deutschen Städte in das europäische Städtesystem als Folge der politischen und vor allem wirtschaftlichen Einigung Europas. Auch diese Entwicklung hat das ehedem stabile arbeitsteilige System der großen westdeutschen Zentren der Nachkriegszeit kräftig durcheinandergewirbelt. Mit der Vollendung des europäischen Binnenmarktes konkurrieren die großen Städte nun nicht mehr nur untereinander, sondern auch mit London, Paris, Mailand, Brüssel und der Randstad Holland um Direktinvestitionen, Unternehmenssitze, Führungskräfte, Touristen, kulturelle Bedeutung und Wissen.

Der dritte Faktor: der Strukturwandel der Wirtschaft. Er lässt sich schlagwortartig beschreiben als Wandel zu einer flexiblen Spezialisierung der Ökonomie, zu einer «flexiblen Netzwerk-Ökonomie». Dieser Wandel ist mit weitreichenden, bisher erst in Konturen erkennbaren Veränderungen in der räumlichen Organisation der Wirtschaft verbunden. Darunter ist ein Prozess besonders wirksam: die zunehmende Schlüsselstellung von ökonomischen Steuerungs-, Dienstleistungs- und Finanzfunktionen anstelle der früher dominierenden industriellen Fertigung. Die «Entindustrialisierung» führt die großen Städte zu einer Wegscheide: Entweder gelingt ihnen der Wandel zu Zentren der «neuen Stadtökonomie» mit Steuerungs-, Dienstleistungs- und Finanzfunktionen, oder sie bleiben krisengeschüttelt zurück.

Der vierte Faktor: der Bedeutungsverlust der Natio- nalstaaten. Zwar übertreibt der gerade im internationalen Business viel gelesene japanische Consulter Kenichi Ohmae sicherlich, wenn er in seinem so betitelten Buch das «Ende des Nationalstaates» proklamiert (Ohmae 1995). Aber seine These, dass in Zukunft weniger die herkömmlichen Territorialstaaten, sondern ökonomisch begründete Regionen mit Metropolen als Kerne die wesentlichen Einheiten des Weltsystems bilden werden, hat viel für sich. Richtig ist jedenfalls, dass die traditionelle staatliche Steuerungsform der Nationalstaaten den neuen weltwirtschaftlichen und weltpolitischen Herausforderungen nicht mehr gerecht zu werden vermag. Vielmehr läuft die Entwicklung auf ein komplexes politisches MehrEbenen-System mit mindestens drei Ebenen hinaus: erstens der supranationalen Ebene (z.B. Europäische Union), zweitens der nationalstaatlichen Ebene und drittens der lokal-regionalen Ebene der «local» oder «region states», wie man im angloamerikanischen Sprachraum sagt.

Den fünften Faktor bilden die anhaltende Arbeitsmarktkrise und die damit teilweise zusammenhängende Finanzkrise der öffentlichen Hände. Daraus resultiert eine Umgewichtung zugunsten wirtschaftspolitischer Entwicklungsziele der Städte und Regionen. In den 1970er Jahren geriet die stabile Nachkriegsordnung mit stetigem Wachstum, Vollbeschäftigung, wohlfahrtsstaatlichen Sicherungssystemen usw. in die Krise. Die Rahmenbedingungen der Stadt- und Regionalpolitik änderten sich grundlegend. Um nicht noch tiefer in die Krise zu schlittern, avancierte die kommunale Wirtschaftspolitik zu einem zentralen Feld der Stadtpolitik. Die Städte sehen sich plötzlich gezwungen, sich im rauen Wind des internationalen Standortwettbewerbs zu "positionieren», wie es im Marketing-Jargon heißt. Wer spielt mit in der Weltliga der Metropolen oder in der Europaliga der «Euro-Metropolen»? Wer sind die Aufsteiger und wer die Absteiger?

Der sechste und vielleicht wichtigste Faktor ist die Globalisierung, also die Transformation des Mosaiks der heute gelegentlich anachronistisch noch sogenannten "Volkswirtschaften» zur «einen Weltwirtschaft». Durch die Entgrenzungen sind die früher vielfach noch national segmentierten Gütermärkte heute immer stärker globalisiert; dieselben Produkte sind praktisch überall auf der Welt zu haben, und zwar nahezu überall zu ähnlichen Preisen. Der eine Weltmarkt ist aber nicht nur zu einem Absatzmarkt geworden, sondern auch zu einem Beschaffungsmarkt; "global sourcing» heißt das Stichwort. Den globalisierten Warenmärkten sind die immateriellen Güter gefolgt: das Geld- und Kreditwesen, Versicherungen, Consulting, Dienstleistungen überhaupt. Diese Form des immateriellen, unsichtbaren Handels, bei dem nur noch Informationen und Geldströme bewegt werden, hat sich inzwischen vom 
Warenhandel weitgehend abgekoppelt und wächst mit atemberaubenden Raten. Mit einem gewissen time lag sind den Gütermärkten die Investitionen gefolgt: Die Erde ist heute nicht nur ein Weltverkehrs- und Welthandelsraum, sondern auch ein Investitionsraum geworden. Große, global operierende Unternehmen verabschieden sich aus ihrer angestammten Region und werden $\mathrm{zu}$ «transnationalen» oder gar «multinationalen Unternehmen». Dadurch verschärft sich schließlich auch der Wettbewerb der Standorte: Sie konkurrieren zunehmend weltweit um Investitionen, Wissen und qualifizierte Arbeitskräfte.

Globalisierung bedeutet Intensivierung des Wettbewerbs auf allen Märkten: a) Waren, b) Dienstleistungen und Informationen, c) Produktionsfaktoren wie Kapital, Arbeitskräfte und Wissen, d) Standorte. Aus der internationalen Standortkonkurrenz resultiert ein Zwang zur Angleichung der Politiken (Sozialpolitik, Fiskalpolitik usw.), andererseits entstehen aber auch neue Möglichkeiten für die regionale und lokale Standortpolitik, die sich um Flächen, um die materielle und immaterielle Infrastruktur, um das Wirtschaftsklima sowie um das regionale Human- und Sozialkapital zu kümmern hat.

In der Humangeographie beginnen wir erst allmählich, uns mit den Folgen der Globalisierung auseinanderzusetzen - sowohl wirtschaftlich als auch politisch und kulturell, gewissermaßen in der «Meta-Geographie» unserer Weltwahrnehmung und Weltdeutung. Die heutige Weltwirtschaftsgeographie unterscheidet sich jedenfalls grundlegend von derjenigen vor zwei bis drei Jahrzehnten (und von derjenigen, die uns im alten Erdkundeunterricht vermittelt wurde).

Die Globalisierung als wesentliche Rahmenbedingung der aktuellen Raumentwicklung zu erkennen, bedeutet selbstverständlich nicht, ihre Logik als Sachzwang misszuverstehen und zur Logik des politischen Handelns zu machen. Es geht vielmehr darum, sich kritisch mit der Globalisierung auseinanderzusetzen, und zwar in einem doppelten Sinne: mit der Globalisierung als unabänderlichem Tatbestand und mit der Globalisierung als politischem Diskurs (BECK 1997). Im politischen Diskurs wird die Globalisierung häufig als argumentative Keule missbraucht, indem neoliberale Propheten der Globalisierung als einer gleichsam natürlichen Marktordnung eine Hegemonialstellung gegenüber konkurrierenden normativen Modellen der politischen, sozialen und ökologischen Weltordnung zumessen (GRUPPE vON LISSABON 1997).

Der siebte und letzte Punkt: In Anlehnung an Manuel Castells weist Peter Taylor (2001) auf die Transformation der Raumsemantik (TAYLOR spricht hier irreführenderweise von Raumontologie) im Gefolge der Globalisierung hin. Unsere tradierte Raumvorstellung aus dem Zeitalter der Nationalstaaten und Nationalökonomien war geprägt von Flächen und Territorien. Darauf aufbauend entwickelte sich eine «mosaic social science» mit territorialbezogenen Daten («state-istics») und Aussagen. Dem steht eine neue Raumsemantik entgegen: ein Raum von Flüssen bzw. Strömen und Knoten, also ein Netzwerk-Raum. Darauf aufbauend muss eine neue "network social science» entwickelt werden. Diese Dualität wird aufgezeigt anhand der Wirtschaftszeitung EсоNOMIsT, die ein Netz von Redaktionen unterhält, aber meist territorial (also auf der Grundlage der traditionellen Raumsemantik) berichtet, während wiederum in der Wirtschaftswerbung die globale Ökonomie als Netzwerk (von Unternehmen, Städten) repräsentiert wird.

Die gesellschaftliche Einbettung der Metropolen-Diskurse macht verständlich, dass ihr Verlauf zyklenhaften Auf- und Abschwüngen folgt, gewissermaßen langen Wellen der gesellschaftlichen Aufmerksamkeit.

Der Begriff «Metropole» kommt bekanntlich aus der griechischen Antike und bezeichnete ursprünglich eine «Mutterstadt», deren Stadtrecht auf koloniale Gründungen übertragen wurde. Später wurde der Begriff allgemein für eine Hauptstadt und seit dem Ausgang der Antike im speziellen Sinn für die Hauptstadt einer Kirchenprovinz (als Sitz eines «Metropolitans») verwendet. Während des Mittelalters und der frühen Neuzeit war die Semantik des Metropolenbegriffs eindeutig religiös-kirchlich bestimmt: Metropolen waren Jerusalem und Rom, im oströmischen Kulturkreis auch Byzanz-Konstantinopel.

Seit dem Aufkommen der modernen Nationalstaaten scheint der Metropolenbegriff zunehmend auf die säkularisierte Kultur und die politische Sphäre bezogen worden zu sein. Beispielsweise wurden in der frühen Neuzeit Paris, Lissabon und London, dann auch Wien als Metropolen bezeichnet. Allerdings müssen wir bei der Verwendung des Metropolenbegriffs zwischen verschiedenen Sprachen unterscheiden. Gerade im Deutschen scheint der Metropolenbegriff noch im 19. Jahrhundert die traditionelle kirchliche Konnotation besessen zu haben, so dass hier eher von «Weltstadt» die Rede war, um auf kulturelle, politische oder auch ökonomische Funktionen abzuheben. Hingegen scheint der Bezug auf die Kirchenhierarchie zumindest bei der englischen "Metropolis», möglicherweise auch bei der französischen "Métropole» schon etwas früher verloren gegangen zu sein.

Jedenfalls hatte seit dem Ende des 19. Jahrhunderts der Metropolenbegriff endgültig seine kirchliche Konnotation verloren, und statt dessen wurde er unter dem Eindruck des industriezeitlichen Städtewachstums 
vor allem für besonders große Städte gebraucht. Dabei spielte wahrscheinlich eine entscheidende Rolle, dass die herkömmlichen Begriffe wie "Stadt», "city», "cité», "Großstadt» usw. nicht mehr ausreichten, um die sowohl quantitativ als auch funktional neue Größenordnung, in die in der zweiten Hälfte des 19. Jahrhunderts insbesondere London, Paris, New York, Wien und Berlin hineinwuchsen, angemessen zum Ausdruck zu bringen. Wenn dann gegen Ende des 19. Jahrhunderts Berlin bereits als «Industriemetropole» bezeichnet wurde, so war das zunächst noch metaphorisch gemeint, markiert aber bereits den Bedeutungswandel, indem nun die Industrie als raison d'être der großen Städte gesehen wird. Damit begann die bis heute anhaltende Ökonomisierung des MetropolenDiskurses.

Heute überwiegen in den Metropolen- und WeltstadtDiskursen eindeutig die ökonomischen bzw. politischökonomischen Konnotationen (SASSEN 1994). Dies hängt nicht nur (und wahrscheinlich nicht einmal primär) mit der gewandelten Realität der Städte selbst zusammen, sondern vor allem mit der semantischen Verschiebung der gesellschaftlichen Weltdeutung und Bewertung aufgrund der Ökonomisierung unserer gesellschaftlichen Deutungsdiskurse. Globale Macht und Ansehen werden heute nicht mehr durch Religionen und Ideologien und nur noch sehr bedingt durch politische und militärische Potenz vermittelt, sondern in erster Linie durch Wohlstand und wirtschaftlichtechnische Leistungsfähigkeit.

Metropolen bzw. Weltstädte werden heute von den meisten Autoren als Knotenpunkte der globalisierten Ökonomie, als wirtschaftliche Steuerungs- und Dienstleistungszentralen mit weltweiten Verflechtungen definiert. Schon die Einbeziehung der politischen Sphäre (z.B. anhand des Indikators "Sitz internationaler politischer Organisationen») ist keineswegs selbstverständlich, und über die Rolle der großen Zentren für die Globalisierung der Kultur liegen nur wenige Studien vor. Allerdings scheint sich in jüngster Zeit in den Sozialwissenschaften ein gewisser Trend abzuzeichnen, die ökonomistische Verkürzung der Metropolenund Weltstadt-Diskurse zu überwinden (FEaTHERSTONE \& LASH 1999, KING 1991, 1995). Möglicherweise erfährt im Zuge des "cultural turn" künftig neben den ökonomischen Funktionen auch wieder die kulturelle Bedeutung der Metropolen als Standorte von Medien und «kreativen Berufen», als Produzenten kultureller Symbole und Deutungsangebote sowie als Laboratorien neuer Lebensstile eine neue Aufwertung.

Wenn die These von der Verschiebung der Raumsemantik vom Territorium zum Netzwerk in der Gegenwart stimmt, wird die Aktualität der Metropolen-Diskurse plausibel: Die Weltgesellschaft und
Weltwirtschaft besteht nicht mehr primär aus einem Mosaik von Nationalstaaten und Nationalökonomien, sondern aus einem multiplen Netzwerk, dessen Knoten die global cities und Metropolregionen sind. Als Standorte supranationaler Steuerungs-, Kontroll- und Dienstleistungsfunktionen bilden sie die entscheidenden Knoten der globalen Verkehrs-, Handels- und Informationsströme. Ihr ökonomischer Kern ist der sog. «Finanz-Dienstleistungs-Komplex».

Für die Standortkonzentration des Finanz-Dienstleistungs-Komplexes in den Metropolen kommt den Faceto-face-Kontakten eine entscheidende Bedeutung zu (SASSEN 1995). Hochspezialisierte Dienstleister wie Spezialanwälte, Computer-Spezialisten, Wirtschaftsprüfer, Finanzdienstleister usw., die in dieser Breite und Tiefe nur in den Metropolen anzutreffen sind, ermöglichen sich wechselseitig und anderen Unternehmen und Organisationen Zeitvorteile für die Verfügbarkeit von Informationen. Zwar können Unternehmensverwaltungen auch in nichtmetropolitanen Städten lokalisiert sein, aber Unternehmen auf hochgradig wettbewerbsintensiven und innovativen Märkten nutzen diese Urbanisationsvorteile und tendieren damit zur Konzentration in Metropolen, weil Informationsvorsprünge für sie ein strategischer Wettbewerbsfaktor sind.

\section{Entwicklung und Ansätze der Metropolenforschung}

Die Entwicklung der gesellschaftswissenschaftlichen Metropolenforschung hängt einerseits mit der Entwicklung der Großstadtforschung, andererseits mit der Entwicklung der gesellschaftlichen Metropolendiskurse zusammen. Insofern lassen sich zwei Entwicklungsphasen unterscheiden, deren Grenze ungefähr um 1980 zu datieren ist (Abb. 1). In der älteren Forschung wird der Terminus «Metropole» nur selten explizit verwendet, aber die Entwicklung und Bedeutung sehr großer Städte - beispielsweise "Weltstädte», «Conurbations», «Metropolitan Areas» o.ä. genannt - wird aus unterschiedlichen theoretischen Perspektiven durchaus thematisiert. Die wichtigsten Ansätze lassen sich zu zwei Gruppen zusammenfassen: erstens die morphologischen Ansätze, in deren Fokus die Größe, Dichte, innere Struktur und äußere Abgrenzung der Großstadtregionen stehen, sowie zweitens die funktionalen Ansätze, die sich mit der Zentralität bzw. der arbeitsteilig ausgebildeten Funktion der Städte in Zentren- bzw. Städtesystemen befassen.

Seit den 1980er Jahren erhielt die Metropolenforschung eine neue Qualität. Teils anknüpfend an die älteren Ansätze, vor allem aber neue Impulse aus der breit gefächerten sozio-ökonomischen Regionalund Entwicklungsforschung aufnehmend, entstand ein 
breites Spektrum innovativer theoretischer und empirischer Forschungsansätze, die sich zu fünf Gruppen typisieren lassen (Abb. 1).

Während die beschreibenden, häufig in einer systemanalytischen Terminologie verfassten Ansätze meist an die Städtesystemforschung der 1970er und 1980er Jahre anknüpfen und eher der Grundlagenforschung zuzurechnen sind, versuchen die geopolitischen Ansätze, Antworten auf praktische Fragen der Raumordnungspolitik zu geben. Eine dritte Gruppe von Forschungsansätzen stellt vor allem auf die ökonomischen Funktionen der Metropolen ab, wobei die Autoren teils mit dem neoklassischen Theorienvorrat der Stadt- und Regionalökonomie, teils mit institutionentheoretischen Ansätzen operieren. In diesen Kontext gehören auch die viel beachteten Arbeiten von SASKIA SASSEN zu den global cities. Eine große vierte Gruppe von Arbeiten argumentiert in einem polit-ökonomischen Theoriehorizont. Dazu gehören sowohl die «Weltsystem-

\begin{tabular}{|c|c|}
\hline \multicolumn{2}{|l|}{ Ältere Ansätze } \\
\hline $\begin{array}{l}\text { Statistisch-beschreibende Ansätze } \\
\text { (z.B. Rank Size Rule) }\end{array}$ & 1910er bis 1970 er Jahre \\
\hline $\begin{array}{l}\text { Sozialökologische Agglomerationsforschung, } \\
\text { insb. innere Stadtstruktur }\end{array}$ & 1920 er bis 1970 er Jahre \\
\hline Zentralörtliche Systeme & 1930 er bis 1980 er Jahre \\
\hline $\begin{array}{l}\text { Ansätze zur Abgrenzung von } \\
\text { Agglomerationsräumen }\end{array}$ & 1950er bis 1990 er Jahre \\
\hline $\begin{array}{l}\text { Städtesystemforschung («Urban } \\
\text { systems») }\end{array}$ & 1960er bis 1990 er Jahre \\
\hline \multicolumn{2}{|l|}{ Neuere Ansätze } \\
\hline $\begin{array}{l}\text { Beschreibende, systemanalytische } \\
\text { Ansätze }\end{array}$ & D. Pumain, N. Cattan, D. Rebitzer \\
\hline Geopolitische Ansätze & R. Brunet, K. Kunzmann \\
\hline $\begin{array}{l}\text { Neoklassisch-institutionenökonomische } \\
\text { Ansätze }\end{array}$ & $\begin{array}{l}\text { P. Hall, S. Sassen, Ch. Jensen- Butler, } \\
\text { R. Camagni, A. Shachar }\end{array}$ \\
\hline \multicolumn{2}{|l|}{ Polit-ökonomische Ansätze } \\
\hline Welthierarchie (Weltsystemtheorie) & $\begin{array}{l}\text { I. Wallerstein, Ch. Chase-Dunn, } \\
\text { P. Taylor }\end{array}$ \\
\hline Globalisierungstheoretische Ansätze & J. Friedmann, R. Cohen, D. Meyer \\
\hline Regulationstheoretische Ansätze & P. Knox, St. Krätke \\
\hline $\begin{array}{l}\text { Globale Hierarchien im «Raum der } \\
\text { Flüsse» }\end{array}$ & $\begin{array}{l}\text { M. Castells, D. Smith, M. Timberlake, } \\
\text { P. Taylor, J. Beaverstock }\end{array}$ \\
\hline Kulturalistische Ansätze & A. King, G. Fuchs \\
\hline
\end{tabular}

Abb. 1: Konzeptionelle Ansätze der Metropolenforschung Conceptual approach to metropolitan research Incitations conceptuelles pour la recherche métropolitaine 
theorie» von Immanuel Wallerstein als auch die sog. World-city-Hypothese von JoHN FriEdmanN und der regulationstheoretische Ansatz von Stefan KRÄTKE. In den letzten Jahren hat die Forschergruppe um Peter TAYLOR und JOHN BEAVERSTOCK der Metropolenforschung wichtige neue Impulse vermittelt, wobei sich eine gewisse Konvergenz von polit-ökonomischen und institutionenökonomischen Theoriebezügen abzeichnet. Weniger mit Strukturen als vielmehr mit Metropolendiskursen befasst sich die Gruppe kulturalistischer Forschungsansätze, die vor allem nach der Rolle der Metropolen im Prozess der sozialen und kulturellen Globalisierung fragen.

Die Metropolenforschung ist heute ein intensiv bearbeitetes Forschungsfeld, das weit über die Stadtforschung hinausreicht und eine Fülle von Querverbindungen zur Wirtschaftsgeographie, Politischen Geographie und zur Raumordnungspolitik aufweist. Ihr Fortschritt verläuft entlang einer aktiven Forschungsfront, an der nicht nur Geographen, sondern auch andere Gesellschafts- und Kulturwissenschaftler in fruchtbarer Kooperation arbeiten.

\section{Das deutsche Raumordnungskonzept der «Europäischen Metropolregionen»}

Die deutsche Raumordnungspolitik hat die strategische Bedeutung von Metropolen lange Zeit ignoriert, übrigens ganz im Gegensatz zu Frankreich und anderen Ländern. Erklären lässt sich dieses Defizit mit der traditionellen Dominanz des Ausgleichsziels, um gleichwertige Lebensbedingungen in allen Teilräumen des Staatsgebiets zu gewährleisten.

Erst 1995 - der Zusammenhang mit der aktuellen Metropolendiskurskonjunktur ist unübersehbar - beschloss die Ministerkonferenz für Raumordnung (MKRO) im «Raumordnungspolitischen Handlungsrahmen» (BUNDESMINISTERIUM 1995) die Ausweisung von sog. «Europäischen Metropolregionen» (EMR) (Michel 1998, 1998a, Ritrer 1997, 1998). Der Handlungsrahmen benennt sechs Metropolregionen: Berlin/ Brandenburg, Hamburg, München, Rhein-Main, RheinRuhr und Stuttgart. In einem weiteren Beschluss vom Juni 1997 bezog dann die MKRO die Stadtregion «Halle/Leipzig-Sachsendreieck» als siebte EMR ein (Abb. 2). Als erstes Bundesland hat Nordrhein-Westfalen diesen Ansatz aufgegriffen und die EMR RheinRuhr förmlich in dem 1995 aufgestellten Landesentwicklungsplan dargestellt und mit landesplanerischen Zielen belegt (LANDESENTWICKLUNGSPLAN 1995).

Die MKRO definiert Europäische Metropolregionen als «räumliche und funktionale Standorte, deren herausragende Funktionen im internationalen Maßstab über die nationalen Grenzen hinweg ausstrahlen» (Bundesministerium 1995). Dann heißt es weiter: «Als Motoren der gesellschaftlichen, wirtschaftlichen, sozialen und kulturellen Entwicklung sollen sie die Leistungs- und Konkurrenzfähigkeit Deutschlands und Europas erhalten und dazu beitragen, den europäischen Integrationsprozess zu beschleunigen.»

Damit wird eine doppelte Zielrichtung der EMR benannt: Das untereinander verflochtene, polyzentrische Netz leistungsfähiger Metropolregionen soll erstens die Wettbewerbsfähigkeit sowohl Deutschlands (auch in Europa) als auch Europas insgesamt in der globalisierten Wirtschaft sichern und zweitens das Zusammenwachsen Europas fördern.

Das EMR-Konzept ist insofern eindeutig entwicklungsorientiert (BloteVogel 1998, 1998a, RITTER 1997, 1998). Vor dem Hintergrund der zunehmenden interregionalen Standortkonkurrenz infolge von Internationalisierung und Globalisierung versuchen Bund und Länder, durch eine Bündelung der auf die einzelnen regionalen Zentren verteilten Entwicklungspotenziale zu einer regional vernetzten Einheit neue Entwicklungschancen zu erschließen. Damit erfolgt eine eindeutige Akzentuierung des Entwicklungsziels gegenüber den anderen beiden Zielen des interregionalen sozialen Ausgleichs und der ökologischen Sicherung innerhalb der bekannten Trias der raumordnungspolitischen Zielsetzungen.

Das EMR-Konzept darf nicht als ein normatives Modell, das eine weitere Vergrößerung der Aktionsräume der Bevölkerung (Arbeits- und Ausbildungspendler, Einkaufsbeziehungen usw.) impliziert, missverstanden werden. Schon die Größe und innere Heterogenität einer EMR wie Rhein-Ruhr oder RheinMain schließen aus, dass die gesamte Region jemals zu einem Lebensraum im Sinne eines homogenen Aktions- und Identifikationsraums der dort lebenden Bevölkerung werden kann. Eine solche Zielsetzung wäre nicht nur unrealistisch, sondern auch mit dem Gebot der Nachhaltigkeit unvereinbar.

Zwar leben die Menschen gerade in mehrkernigen Ballungsräumen wie Rhein-Ruhr und Rhein-Main immer weniger in einzelnen Städten, sondern immer mehr in Regionen, also in Räumen, die weit über die politischen Grenzen einzelner Gemeinden hinausreichen, ohne nach außen eindeutig (und schon gar nicht politisch-administrativ) abgrenzbar zu sein. Aber dennoch bleibt ein wichtiger Mittelpunkt der Lebensbezüge und der raumbezogenen Identifikation kleinräumig, gleichsam als Kombination von Kiez und Region. Gerade mehrpolige Metropolregionen wie Rhein-Ruhr und Rhein-Main bieten beides: dezentrale, kleinräumige Ortsbezogenheit der Lebenswelt und metropolitane Offenheit und Internationalität. 


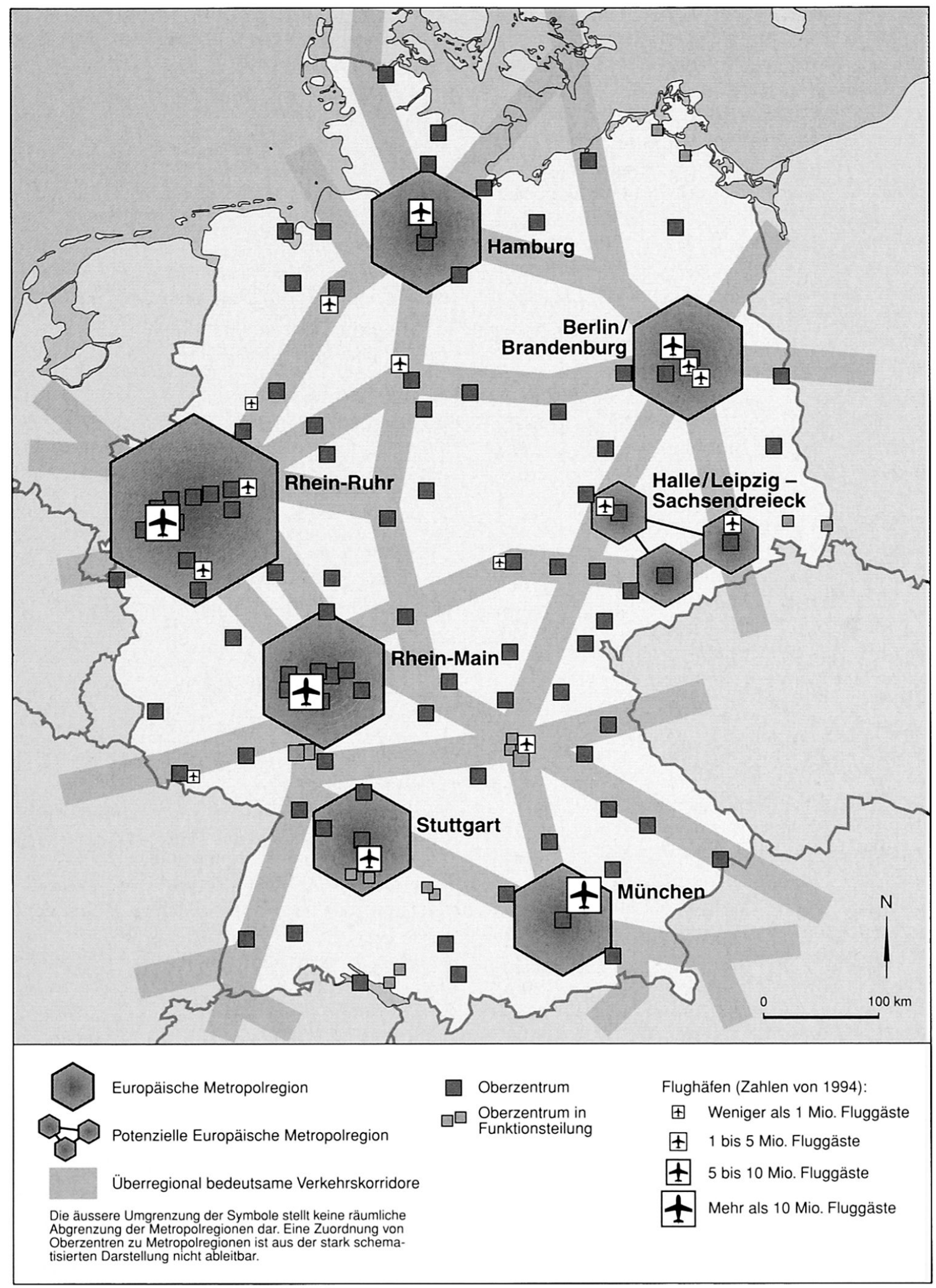

Abb. 2: Europäische Metropolregionen in Deutschland European Metropolitan Regions in Germany

Régions métropolitaines en Allemagne

Quelle: Bundesforschungsanstalt für Landeskunde und Raumplanung 1997, Entwurf der Arbeitsgruppe Europäische Metropolregionen der MKRO; Kartographie: H. KRÄHE 
Eine besonders interessante, aber noch wenig untersuchte Frage bezieht sich auf die Frage, ob die funktionale und politische Vielkernigkeit von Metropolregionen wie Rhein-Ruhr, Rhein-Main und Randstad Holland eher ein Handicap oder gerade ein besonderes Entwicklungspotenzial darstellt. Eine vorläufige Abwägung der Argumente legt den Schluss nahe, dass insgesamt gesehen (und basierend auf der bisherigen Entwicklung) die ökonomischen Vorteile einer monozentrischen Ballung überwiegen, während eine polyzentrische Metropolregion eher ökologische Vorteile besitzt und bessere Chancen hat, den Anforderungen einer nachhaltigen Stadt- und Regionalentwicklung gerecht zu werden.

Was bedeutet die landesplanerische Funktionsbestimmung einer Stadtregion als Metropolregion für die lokale und regionale Politik? Klarer als eine positive Antwort ist zunächst eine negative: Eine Metropolregion ist keine neue, zusätzliche Verwaltungsebene. Das EMR-Konzept zielt auf etwas anderes, nämlich auf eine neue Sichtweise der Region als Ganzes, auf eine entwicklungspolitische Koordinierung der Handlungen der relevanten Akteure und speziell auf eine Mobilisierung und Bündelung von vorhandenen, aber bisher stark zersplitterten Ressourcen. Um solche Ziele zu erreichen, ist eine gewisse formelle Verankerung in den landesplanerischen Programmen und Plänen zwar notwendig, aber bei weitem nicht hinreichend. Entscheidend ist vielmehr eine Implementierung auf der politischen Handlungsebene.

Die konkrete Umsetzung des raumordnungspolitischen Konzepts der Europäischen Metropolregionen ist in den einzelnen Regionen Deutschlands sehr unterschiedlich weit. In Berlin-Brandenburg versucht eine gemeinsame Landesplanung zwar ihr Bestes, doch wirkt sich das Scheitern der Länderfusion hemmend aus. Am weitesten sind die Metropolregionen Hamburg und Stuttgart, während in der Rhein-MainRegion zwar seit einigen Jahren eine intensive Diskussion stattfindet, ohne bisher jedoch zu wirksamen Lösungen geführt zu haben. Im Jahre 2000 hat sich eine lockere Arbeitsgemeinschaft der Metropolregionen gebildet, in der Analyse-, Strategie- und Implementationsfragen behandelt werden.

Für die Europäische Metropolregion Rhein-Ruhr hat der nordrhein-westfälische Landesentwicklungsplan von 1995 die Zielsetzungen des Handlungsrahmens der MKRO in geltendes Recht übernommen, und die seitdem novellierten Gebietsentwicklungspläne der einzelnen Teilregionen greifen diese Perspektive auf und konkretisieren sie weiter. Auf einer Konferenz der Planungsamtsleiter des Ruhrgebiets wurde das Konzept ausdrücklich begrüßt und als Ausgangspunkt für eine strategische Ausrichtung der Stadtplanungen der beteiligten Städte akzeptiert.

Allerdings sind die großen Schwierigkeiten unübersehbar, die einer Umsetzung dieses Konzepts in praktisches politisches und planerisches Handeln gegenüberstehen. Es genügt keinesfalls, das Konzept als landesplanerisches Ziel von oben vorzugeben, entscheidend für die praktische Wirkung ist, inwieweit auch die Städte und Kreise sowie die anderen für den Prozess der Regionalentwicklung bedeutsamen Akteure wie Kammern von der neuen Perspektive überzeugt und bereit sind, ihr Handeln daran auszurichten. Die Internationale Bauausstellung EMSCHER PARK hat hier durchaus beispielgebend gewirkt.

Die Erfahrung zeigt allerdings auch, dass es gerade in mehrpoligen Metropolregionen wie Rhein-Ruhr außerordentlich schwierig ist, die Städte zu kooperativem Handeln zu bewegen. Während in monozentrischen Metropolregionen mit einer großen Kernstadt deren politisch-administrative Führung i.d.R. die Rolle des strategischen «Prozess-Promotors» übernimmt (allerdings mit dem Folgeproblem, dass sich die Umlandkreise oft dominiert fühlen), muss diese Rolle bei großen mehrpoligen Gebilden entweder von der übergeordneten staatlichen Ebene oder aber von einer kommunalpolitischen Führungsgruppe (z.B. Konferenz der Oberbürgermeister) übernommen werden. Klar ist jedenfalls, dass solche Prozesse nicht quasi automatisch durch «Selbstorganisation» vorankommen, sondern einer treibenden Kraft und positiver Anreize von Seiten der Länder und des Bundes bedürfen.

\section{Bewertung}

Die Entwicklung von Metropolen steht seit einigen Jahren auch in Deutschland auf der politischen Agenda. In der deutschen Raumordnungspolitik steht dafür das Konzept der «Europäischen Metropolregionen». Wie ist diese Innovation einzuordnen und zu bewerten?

Dieser neue raumordnungspolitische Diskurs ist eng verknüpft mit den allgemeineren gesellschaftlichen Diskursen über Metropolen einerseits sowie Europäisierung und Globalisierung andererseits. Nach allem, was wir über die «Geographie» der globalisierten «neuen Ökonomie» wissen, nimmt die Bedeutung der Metropolen nicht ab, sondern zu. Als Knotenpunkte der globalen Netzwerk-Ökonomie sind sie gewissermaßen deren räumliches Skelett geworden, das insofern funktional äquivalent an die Stelle der tradierten territorialen Ordnung von Nationalstaaten und Volkswirtschaften getreten ist. Die neue Weltwirtschaft ist nicht einfach entgrenzt und enträumlicht, sondern 
führt zu einer neuen "Geographie», deren Konturen sich allerdings bisher nur in Umrissen abzeichnen.

Wenn diese Diagnose stimmt, bekommt die Frage nach «Metropolen in Deutschland» eine neue politische Relevanz, und zwar jenseits lokal- und regionalpolitischer Eitelkeiten und Marketing-Strategien. Wenn im globalen Wettbewerb um Investitionen, Innovationen, Wissen und hochqualifizierte Arbeitskräfte die Standortqualitäten von Metropolregionen künftig den Ausschlag geben, kann es weder den Staaten noch den betreffenden Städten gleichgültig sein, inwieweit das jeweilige urbane bzw. metropolitane System leistungsund wettbewerbsfähig ist.

Können diese Funktionen nur von einer einzigen Metropole erfüllt werden? Braucht Deutschland also «wieder» eine metropolitane Spitze - so wie Frankreich, Großbritannien und Japan - und so, wie es in den ersten vier Jahrzehnten des 20. Jahrhunderts einmal war? Die Erwartung - sei es Hoffnung oder Befürchtung -, dass Berlin künftig wieder diese Rolle übernehmen würde, ist aus verschiedenen Gründen obsolet: Erstens ist sie völlig unrealistisch, weil die historische Verlagerung der metropolitanen Funktionen auf die großen Zentren im westlichen Deutschland auf lange Sicht nicht rückgängig zu machen ist. Zweitens verkennt diese Erwartung, dass der föderale Staatsaufbau und das Prinzip einer regional ausgewogenen Entwicklung Deutschlands mit der Vorstellung einer übermächtigen, riesenhaften Metropole unvereinbar sind. Und drittens ist diese Erwartung auch unter entwicklungspolitischen Gründen obsolet, denn die Leistungs- und Wettbewerbsfähigkeit von Städtesystemen hängt keineswegs von der Existenz einer einzigen überragenden Metropole ab, sondern von der Standortqualität und einer effizienten Arbeitsteilung und Vernetzung der polyzentrischen Siedlungsstruktur. In Deutschland kommt insofern der gesamten Gruppe der Metropolregionen - Berlin, Hamburg, Rhein-Ruhr, Rhein-Main und München, möglicherweise ergänzt um Stuttgart sowie Halle/Leipzig-Sachsendreieck - eine besondere Rolle zu. Deutschland hat und braucht nicht eine Metropole, sondern ein metropolitanes Netzwerk als integraler Bestandteil des europäischen Städtesystems.

Mit der Thematisierung der metropolitanen Funktionen und der Einführung der planerischen Kategorie der EMR hat in der deutschen Raumordnungspolitik eine deutliche Problemverschiebung stattgefunden. Die Raumordnung hat sich von ihrer traditionellen Fixierung auf interregionalen Disparitätenausgleich und Fragen der Ressourcenverteilung gelöst und unter dem Eindruck von Europäisierung und Globalisierung die Entwicklungsfunktion der großen Zentren in den Fokus der Politik gerückt. Sie hat damit relativ spät nachvollzogen, was auf der kommunalen Ebene schon seit den 1980er Jahren als Bedeutungsgewinn der kommunalen Wirtschaftspolitik zu beobachten ist.

Insofern signalisiert der Wandel der Repräsentation der großen Zentren in den Plänen und Programmen der deutschen Raumordnung einen Wandel der strategischen Ausrichtung. In der traditionellen Raumordnungspolitik der 1960er bis 1980er Jahre wurde die primäre Funktion der großen Städte in der Bereitstellung von Ressourcen (Güter, Dienste, Arbeitsplätze) für ihre jeweiligen Verflechtungsbereiche gesehen; dem entsprach ihre Repräsentation in den Plänen und Programmen als Zentrale Orte und Schnittpunkte von Entwicklungsachsen. Mit der Einführung des Raumbildes der «Europäischen Metropolregion» rücken die Entwicklungs-, Wettbewerbs- und Innovationsfunktionen der großen Stadtregionen und ihre Funktion als Knoten der europäischen und globalisierten NetzwerkÖkonomie in den strategischen Fokus der Politik. Dabei lenkt die Formulierung «Metropolregion» den Blick auf die Tatsache, dass Metropolen heute immer weniger dem tradierten Modell der kompakten Stadt in ihren kommunalen Grenzen entsprechen, sondern sich zu mehrkernigen Gebilden - sei es als StadtUmland-Verbund (Beispiel Berlin-Brandenburg), sei es als regionaler Städte-Cluster (Beispiel Rhein-Ruhr) - entwickeln. Der Terminus «Metropolregion» ist insofern als ein neues strategisches Raumbild zu verstehen, mit dem die deutsche Raumordnungspolitik auf veränderte Anforderungen reagiert und das zugleich ein verändertes Selbstverständnis der Planung signalisiert.

Sich mit Metropolisierungs- und Globalisierungsdiskursen wissenschaftlich $\mathrm{zu}$ befassen, bedeutet keineswegs, deren normative Implikationen kritiklos zu übernehmen. Sonst wird aus diesen Diskursen flugs ein vermeintlicher Sachzwang, demgegenüber Erfordernisse der interregionalen Verteilungsgerechtigkeit und der Sicherung der natürlichen Lebensgrundlagen auf der Strecke bleiben. Es kann deshalb in der heutigen Raumordnungspolitik auch weder um eine Apologie der Logik des Weltmarktes noch um ein starres Festhalten an tradierten Wahrnehmungs- und Handlungsmustern, das die neuen Herausforderungen ignorieren zu können glaubt, gehen. Metropolenorientierte Stadt- und Regionalpolitik bedeutet nicht eine bedenkenlose Ausrichtung auf die tatsächlichen oder vermeintlichen Anforderungen der Globalisierung, sondern eine aktiv gestaltende Politik, die sich an den Interessen und Bedürfnissen der Bevölkerung orientiert. Die Leitvorstellung der «nachhaltigen Entwicklung», inzwischen an prominenter Stelle im Baugesetzbuch und im Raumordnungsgesetz der Bundesrepublik Deutschland verankert, bietet dafür einen angemessenen Orientierungsrahmen. 


\section{Literatur}

Beaverstock, J.V., R.G. Smith \& P.J. TAylor (1999): A roster of world cities. - In: Cities 16: 445-458.

Beaverstock, J., R.G. Smith \& P.J. TAylor (2000): World-city net-works: a new metageography? - In: Annals of the Association of American Geographers 90: 123-134.

BECK, U. (1997): Was ist Globalisierung? Irrtümer des Globalismus - Antworten auf Globalisierung. - = Edition Zweite Moderne, Frankfurt a.M.: Suhrkamp.

Blotevogel, H.H. (1998): Europäische Metropolregion Rhein-Ruhr. Theoretische, empirische und politische Perspektiven eines neuen raumordnungspolitischen Konzepts. - = ILS-Schrift 135, Dortmund: Institut für Landes- und Stadtentwicklungsforschung des Landes Nordrhein-Westfalen.

Blotevogel, H.H. (1998a): Metropolen als Motoren der Raumentwicklung und als Gegenstand der Raumordnungspolitik. - In: Deutschland in der Welt von morgen, Hannover: ARL, = Forschungs- und Sitzungsberichte 203: 62-70.

BRUNET, R. et al. (1989): Les villes européennes. - Rapport pour la DATAR, Hrsg.: Groupement d'Intérêt Public RECLUS, Paris: La Documentation Française. Bundesministerium Für RaUmoRdNung, BaUwESEN UND STÄDTEBAU (Hrsg.) (1995): Raumordnungspolitischer Handlungsrahmen. - Bonn: BMBau.

Camagni, R. \& T. Pompili (1990): Competence, power and waves of urban development: an Italian example. - In: NiJKamP, P. (ed.): Sustainability of urban systems. - Aldershot: Avebury: 37-86, = Avebury Urban Europe Series.

Castells, M. (1996/97): The information age: Economy, society and culture. -3 Bde., Oxford: Blackwell. Bd. 1: The rise of the network society (1996), Bd.2: The power of identity (1997), Bd. 3: End of the millennium (1997).

Cattan, N., Pumain, D., Rozenblat, C. \& T. SaintJulien (1994): Le système des villes européennes. Collection VILLES, Paris: Anthropos.

Chase-Dunn, C.K. (1985): The system of world cities, A.D. 800-1975. - In: Timberlake, M. (ed.): Urbanization in the world-economy. - Orlando, Fl.: Academic Press: $269-292$, = Studies in social discontinuity.

Cohen, R.B. (1981): The new international division of labor, multinational corporations and urban hierarchy. - In: DeAR, M. \& A.J. ScotT (eds): Urbanization and urban planning in capitalist society. - London: Methuen: 287-315.

FALUdI, A. (1996): Framing with images. - In: Environment and Planning B: Planning and Design 23: 93-108. Faludi, A. (1998): From planning theory mark 1 to planning theory mark 3. - In: Environment and Planning B: Planning and Design 25: 110-117.

FAludi, A. \& VAN DER FAlK, A. (1994): Rule and order. Dutch planning doctrine in the twentieth century. Dordrecht: Kluwer.
Featherstone, M. \& S. Lash (eds) (1999): Spaces of culture. City, nation, world. - London: Sage.

FriedmanN, J. (1986): The world city hypothesis. - In: Development and Change 17:69-83.

Fuchs, G., Moltmann, B. \& W. Prigge (Hrsg.) (1995): Mythos Metropole - Frankfurt a.M.: Suhrkamp, = Edition Suhrkamp 1912.

FüRST, D. (1997): Wandel raumplanerischer Leitbilder - Wandel raumplanerischen Denkens. - In: Monheim, H. \& C. ZÖPEL (Hrsg.): Raum für Zukunft. - Essen: Klartext: 108-122.

Grunwald, A. (1996): Kulturalistische Planungstheorie. - In: HartmanN, D. \& P. JANISCH (Hrsg.): Methodischer Kulturalismus. - Frankfurt a.M.: Suhrkamp: 315-345.

GRUPPE VON LisSABON, DIE (Hrsg.) (1997): Grenzen des Wettbewerbs. - München: Luchterhand. (Orig.: Group of Lisbon: Limits of competition. - Cambridge, Mass.: MIT Press 1995).

HAJER, M.A. (2000): Transnational networks as transnational policy discourse: Some observations on the politics of spatial development in Europe. - In: SALET, W. \& A. FALUdi (Hrsg.): The revival of strategic spatial planning. - Amsterdam: Royal Netherlands Academy of Arts and Sciences: 135-142.

Hall, P. (1998): Cities in civilization. Culture, innovation and urban order. - London: Weidenfeld \& Nicolson.

IPSEN, D. (1997): Raumbilder. Kultur und Ökonomie räumlicher Entwicklung. - = Stadt, Raum und Gesellschaft 8, Pfaffenweiler: Centaurus.

Jensen-Butler, C., Shachar, A. \& J. van Weesep (eds) (1997): European cities in competition. - Aldershot: Avebury.

KING, A.D. (1991): Urbanism, colonialism and the world economy. Cultural and spatial foundations of the world urban system. - London: Routledge.

KING, A.D. (1995): Re-presenting world cities: cultural theory/social practice. - In: KNOX, P.L. \& P.J. TAYLOR (eds): World cities in a world-system. - Cambridge: Cambridge University Press: 215-231.

Knieling, J. (2000): Leitbildprozesse und Regionalmanagement. Ein Beitrag zur Weiterentwicklung des Instrumentariums der Raumordnungspolitik. - = Beiträge zur Politikwissenschaft 77, Frankfurt a.M.: Lang.

KNOX, P.L. (1995): World cities in a world-system. In: KNox, P.L. \& P.J. TAYLOR (eds): World cities in a world-system. - Cambridge: Cambridge University Press: 3-20.

KRÄTKE, S. (1991): Strukturwandel der Städte. Städtesystem und Grundstücksmarkt in der "post-fordistischen» Ära. - Frankfurt: Campus.

KrätKe, S. (1992): Hierarchie und Vernetzung im europäischen Städtesystem. - = FSP 1-06 Stadtökonomie, Diskussionsbeitrag 13, Hamburg: TU HamburgHarburg. 
KRÄTKE, S. (1998): Globalisierung und Stadtentwicklung in Europa. - In: Geographische Zeitschrift 85: 143-158.

KunZmann, K.R. (1992): Zur Entwicklung der Stadtsysteme in Europa. - In: Mitteilungen der Österreichischen Geographischen Gesellschaft 134: 25-50.

LACKNER, M. \& M. WeRner (Hrsg.) (1999): Der cultural turn in den Humanwissenschaften. Area Studies im Auf- und Abwind des Kulturalismus? - = Werner Reimers Konferenzen, Schriftenreihe Suchprozesse für innovative Fragestellungen in der Wissenschaft 2, Bad Homburg: Werner Reimers Stiftung.

LANDESENTWICKLUNGSPLAN NORDRHEIN-WESTFALEN (LEP NRW) vom 11.5.1995 (1995). - In: Gesetz- und Verordnungsblatt NRW 49, Nr. 50:532-565.

MATEJovSKI, D. (Hrsg.) (2000): Metropolen. Laboratorien der Moderne. - = Schriften des Wissenschaftszentrums NRW 5, Frankfurt a.M.: Campus.

MEYER, D.R. (1986): The world system of cities: relations between international financial metropolises and South American cities. - In: Social Forces 64: 553-581. Michel, D. (1998): Das Handlungskonzept der Ministerkonferenz für Raumordnung für europäische Metropolregionen und seine Umsetzung. - In:Deutschland in der Welt von morgen, Hannover: ARL, = Forschungs- und Sitzungsberichte 203: 55-61.

Michel, D. (1998a): Das Netz der europäischen Metropolregionen in Deutschland. Raumordnungspolitische Fragestellungen an die Regional- und Raumforschung. - In: Raumforschung und Raumordnung 56: 362-368. OHMAe, K. (1995): The end of the nation state. The rise of regional economies. - New York: Free Press.

PLøGER, J. (2001): Millennium urbanism - discursive planning. - In: European Urban and Regional Studies 8: 63-72.

ReBITZER, D.W. (1995): Internationale Steuerungszentralen. Die führenden Städte im System der Weltwirtschaft. $-=$ Nürnberger Wirtschafts- u. Sozialgeographische Arbeiten 49, Nürnberg: Wirtschafts- und Sozialgeographisches Institut der Universität.

RitTer, E.-H. (1997): Europäische Metropolregion Rhein-Ruhr oder: Kann aus einer Städte-Agglomeration eine Metropolregion werden? - In: Räumliche Disparitäten und Bevölkerungswanderungen in Europa, Hannover: Akademie für Raumforschung und Landesplanung, = ARL Forschungs- und Sitzungsberichte 202: 156-170.

RitTER, E.-H. (1998): Metropolen im supranationalen Wettbewerb. - In: Deutschland in der Welt von morgen, Hannover: Akademie für Raumforschung und Landesplanung, = ARL Forschungs- und Sitzungsberichte 203: 51-54.

SALET, W. \& A. FALUdi (eds) (2000): The revival of strategic spatial planning. - Amsterdam: Royal Netherlands Academy of Arts and Sciences, = Verhandelingen/ Koninklijke Nederlandse Akademie van Wetenschappfen, Afd. Letterkunde, Nieuwe Reeks, deel 181.
SASSEN, S. (1991): The global city: New York, London, Tokyo. - Princeton, N.J.: Princeton University Press. SASSEN, S. (1994): Cities in a world economy. - Thousand Oaks, Cal.: Pine Forge.

SASSEN, S. (1995): On concentration and centrality in the global city. - In: KNOX, P.L. \& P.J. TAYLOR (eds): World cities in a world-system. - Cambridge: Cambridge University Press: 63-75.

SASSEN, S. (1995a): Metropole, Grenzen eines Begriffes. - In: Fuchs, G., Moltmann, B. \& W. Prigge (Hrsg.): Mythos Metropole. - Frankfurt a.M.: Suhrkamp: 165-177.

Smith, D. M. (1996): Third world cities in global perspective: the political economy of uneven urbanization. - Boulder, Col.: Westview Press.

TAYLOR, P.J. (1995): World cities and territorial states: the rise and fall of their mutuality. - In: KNox, P.L. \& P.J. TAYLOR (eds): World cities in a world-system. Cambridge: Cambridge University Press: 48-62.

TAYLOR, P.J. (1997): Hierarchical tendencies amongst world cities: a global research proposal. - In: Cities 14: 323-332.

TAYLOR, P.J. (2001): Being economical with the geography. Global and World Cities Study Group and Network, Research Bulletin 39. - http://www.lboro.ac.uk/ departments/gy/research/gawc/rb/rb39.html

THORnLey, A. (2000): Strategic planning in the face of urban competition. - In: SALET, W. \& A. FALUdi (eds): The revival of strategic spatial planning. - Amsterdam: Royal Netherlands Academy of Arts and Sciences: 39-51.

Timberlake, M. (ed.) (1985): Urbanization in the world-economy. - Orlando, Fl.: Academic Press.

WALLERSTEIN, I. (1974/80/89): The modern world-system. - New York: Academic Press. Vol. 1: Capitalist agriculture and the origins of the European worldeconomy in the 16th century (1974), Vol. 2: Mercantilism and the consolidation of the European worldeconomy, 1600-1750 (1980), Vol. 3: The second era of great expansion of the capitalist world-economy, 1730-1840s (1989).

ZONNEFELD, W. (2000): Discursive aspects of strategic planning. A deconstruction of the «balanced competitiveness» concept in European spatial planning. In: SALET, W. \& A. FAludi (eds): The revival of strategic spatial planning. - Amsterdam: Royal Netherlands Academy of Arts and Sciences: 267-282.

\section{Zusammenfassung: Die Metropolregionen in der Raumordnungspolitik Deutschlands - ein neues strategisches Raumbild?}

Mitte der 1990er Jahre wurde die neue raumordnungspolitische Kategorie der «Europäischen Metropolregion» (EMR) in die Raumordnungspolitik der Bundesrepublik Deutschland eingeführt. In dem Aufsatz wird die Frage diskutiert, inwieweit damit ein neues 
strategisches Raumbild verwendet wird, das mit einer normativen Umorientierung der Raumordnungspolitik verbunden ist. Diese Frage wird eingebettet in den weiteren Diskurskontext über Metropolen, der seit einigen Jahren nicht nur in der Wissenschaft, sondern auch in der Politik und den Medien eine auffällige Konjunktur erlebt. Angesprochen werden sowohl die Hintergründe dieser Diskurskonjunktur wie auch die Entwicklung der Metropolenforschung. Die Analyse der Zielrichtung des raumordnungspolitischen Konzepts der Europäischen Metropolregion zeigt, dass die Einführung der Metropolen-Semantik in die deutsche Raumordnungspolitik zu verstehen ist als Reaktion auf eine ganze Reihe von externen Herausforderungen. Sie führt zu einer tiefgreifenden Verschiebung des raumordnungspolitischen Selbstverständnisses, indem sich die Raumordnung von ihrer traditionellen Fixierung auf interregionalen Disparitätenausgleich und Fragen der Ressourcenverteilung und Ressourcensicherung löst und unter dem Eindruck von Europäisierung und Globalisierung die Entwicklungsfunktion der großen Zentren in den Fokus der Politik rückt.

Summary: Metropolitan Regions in German Regional Policy - a new and strategic phenomenon?

During the mid-1990's, a new politically significant term was introduced into the Regional Policy of the Federal Republic of Germany: the «European Metropolitan Region (EMR)». This paper focuses on the ongoing discussion regarding the degree to which this new strategic regional term owes its creation to a normative re-orientation of local regional policy. Further, it looks at the general upswing of interest taken in metropolitan discourse in recent years, not only in the sciences, but in politics and the media as well. Background information regarding the upsurge of interest in this field is given, and the development of metropolitan research is traced. The analysis of the objectives set by the regional policy of European Metropolitan Regions indicates that the introduction of metropolitan semantics into German Regional Policy is the reaction to a number of external challenges. This step has led to a profound shift in regional policy attitudes. Due to the pressures exerted by Europeanisation and globalisation, regional policy moved away from traditional fixation on interregional disparity compensation and resource distribution and placed the concern for major centre development into the political arena.
Résumé: Les régions métropolitaines dans la politique d'aménagement du territoire en Allemagne Une nouvelle image stratégique de l'espace?

Au milieu des années 1990 la politque d'aménagement du territoire de la République Fédérale d'Allemagne a intégré en tant que concept spatio-politique la nouvelle notion de "région métropolitaine européenne» (EMR: «Europäische Metropolregion»). Dans la présente contribution la discussion porte sur la question dans quelle mesure il est fait appel ici à une image stratégique spatiale liée à une réorientation normative de la politique d'aménagement du territoire. Cette question est insérée en outre au contexte discursif sur les métropoles qui est depuis quelques années en vogue non seulement en science, mais aussi en politique et dans le monde médiatique. Sont interrogées tant les raisons de ce discours conjoncturel que l'évolution de la recherche métropolitaine. L'analyse des objectifs de ce concept d'aménagement de la région métropolitaine européenne montre que l'introduction de la sémantique européenne dans la politique d'aménagement du territoire allemande est à interpréter comme une réaction à toute une série d'interpellations externes. Il s'agit là d'un passage de l'image de l'aménagement telle qu'elle a été perçue en soi à travers la fixation traditionnelle sur la recherche de l'élimination des disparités interrégionales ainsi que sur la répartition des ressources, vers une focalisation politique sur la fonction de développement des grands centres sous l'éclairage de l'européanisation et de la globalisation.

Prof. Dr. Hans Heinrich Blotevogel, G.-MercatorUniversität Duisburg, Institut für Geographie, Lotharstrasse 65, D-47057 Duisburg.

e-mail: blotevogel@uni-duisburg.de

Manuskripteingang/received/rentrée du manuscrit: 9.10.2001

Annahme zum Druck/accepted for publication/accepté pour l'impression: 5.11.2001 